\title{
Ultrashort Pulse Laser Processing of Transparent Materials
}

\author{
Fumiyo YOSHINO, Haibin ZHANG and Alan ARAI \\ IMRA America, Inc., Applications Research Laboratory \\ 48834 Kato Road, Suite 106A, Fremont, CA 94538 U.S.A. \\ E-mail: fyoshino@imra.com
}

\begin{abstract}
Ultrashort pulse lasers can generate extremely high peak power with a modest average power. For example, a 1-W average power laser can produce pulses with a peak power of $20 \mathrm{MW}$ (500 fs, $100 \mathrm{kHz}$ ). Focusing this extremely high peak power laser beam enables a range of material modifications in and on transparent materials. Fiber lasers can produce micro Joule pulses at repetition rates from the $100 \mathrm{~s}$ of $\mathrm{kHz}$ to the $\mathrm{MHz}$ range.

Sub-surface optical waveguides written by ultrashort pulse lasers in a range of transparent materials have been reported on in the past. By adjusting the focusing conditions, pulse energy and other laser processing parameters, it is possible to create well-defined material modifications or even cracks below the surface. These features can be used to produce different types of marks and icons. By properly controlling the processing conditions, it is possible to produce features that are relatively difficult to see under normal, ambient lighting, but clearly visible when illuminated from the edge - the Visibility Trade-Off.

DOI: $10.2961 / \mathrm{jlmn} .2009 .03 .0013$
\end{abstract}

Keywords: femtosecond, ultrashort pulse, micromachining, marking, glass, polycarbonate

\section{Introduction}

In recent years, ultrashort pulse lasers have gradually started to be adopted by industries outside of the research lab (e.g. wafer dicing, LASIK, etc.). One of the unique machining characteristics of ultrashort pulse lasers is a much reduced thermal effect for cleaner processed features since the energy is deposited in a time duration similar to or shorter than thermal diffusion time [1]. An ultrashort pulse also offers higher efficiency for energy absorption in transparent materials via a nonlinear process, which is a function of the intensity of the laser pulse [2]. This process produces machined features that are significantly more localized compared to the case with longer duration pulses.

IMRA's FCPA $\mu$ Jewel ultrashort pulse fiber laser system offers a useful combination of high energy and high repetition rate, which is required for fast processing in industrial manufacturing.

High repetition rate is not only advantageous for faster process time, but it can also be used to manage thermal effects during laser processing. How much energy is deposited in the material, how long it takes for the energy to diffuse out of the affected volume, and when and where the next pulse is deposited controls the overall spatio-temporal temperature evolution. Depending on the material's thermal and mechanical properties and the laser process parameters, different material modifications can be produced. In some cases, a controlled thermal effect is needed. Such applications include optical waveguide writing [3] and transparent material welding $[4,5,6]$.

The FCPA $\mu$ Jewel D-1000 produces an average power of $1 \mathrm{~W}$, approximately constant over a repetition rate range from $100 \mathrm{kHz}$ to $5 \mathrm{MHz}$. A pulse energy up to $10 \mu \mathrm{J}$ is available at the lowest repetition rate, $100 \mathrm{kHz}$. IMRA's FCPA $\mu$ Jewel system is designed to be a reliable and robust laser, with units exceeding 18,000 hours of operation. Figure 1 shows a photo of the laser head and controller.

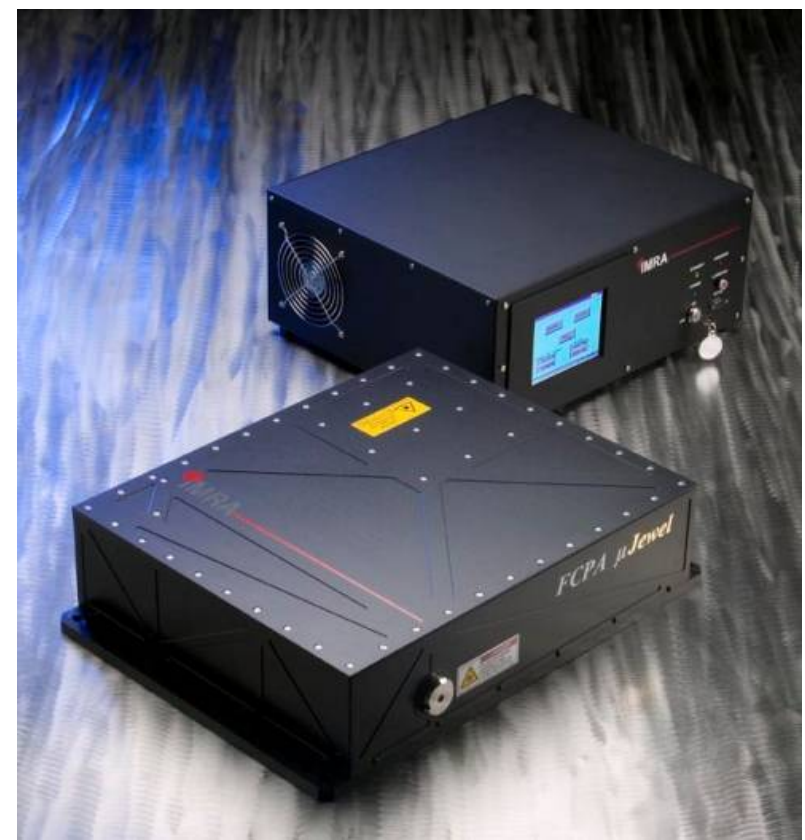

Fig. 1 FCPA $\mu$ Jewel D-1000 laser head \& controller.

The laser head has compact dimensions of $550 \mathrm{x} 428 \mathrm{x}$ $131 \mathrm{~mm}^{3}$ and weighs about $30 \mathrm{~kg}$. It is an ideal system for $24 / 7$ operation in a variety of manufacturing environments.

By controlling process parameters such as power, repetition rate, focusing geometry and scanning speeds, a wide variety of materials can be modified to produce different features $[7,8]$.

In this paper, we present a unique micro-marking feature, Switchable Inner Micro-Marking (SwIMM), in vari- 
ous industrially important transparent materials using IMRA's FCPA $\mu$ Jewel D-1000 ultrashort pulse fiber laser.

\section{Switchable Inner Micro-Mark (SwIMM)}

SwIMM are a type of sub-surface material modification within transparent materials produced by high repetition rate, ultrashort laser pulses.

Subsurface material modification is possible because of the efficient nonlinear absorption process, which takes place only in the region of the focal volume. Ultrashort pulses also produce features that are very precisely localized because less energy is needed to achieve a threshold amount of absorption compared to longer pulses, significantly reducing unwanted and uncontrolled thermal effects. These characteristics offer better control of the mark size and shape.

With IMRA's FCPA $\mu$ Jewel ultrashort pulse fiber laser system, the repetition rate can be changed from $100 \mathrm{kHz}$ to $5 \mathrm{MHz}$ with pulse energy up to $10 \mu \mathrm{J}$ (at $100 \mathrm{kHz}$ ). Process speed, focusing condition, pulse energy, and repetition rate can be widely adjusted to control the thermal process and create marks within various transparent materials.

\subsection{Visibility Trade-Off}

SwIMM is unique among other subsurface marks in transparent materials because the visibility contrast between the ON and OFF states is very high. In ambient lighting, the marks are not very visible to the unaided eye, almost as if there is no mark (OFF state). With an external illumination source directed at the mark, such as an LED or laser diode, the mark's visibility can be dramatically increased (ON state). This visibility trade-off makes it a "switchable" mark by controlling the illumination source. In its OFF state, the object can be used as a normal transparent material. In the ON state, necessary information can be effectively delivered by the SwIMM to the viewers.

This unique switchable mark with controlled visibility trade-off can be adapted to different application areas.

\section{SwIMM Types and Application Examples}

Several different types of SwIMM have been made in different materials. Scattering SwIMM are visible when the illumination light is scattered from the laser-induced material modifications. Reflective SwIMM are visible when the illumination light is reflected from the laser-induced material modification towards the viewer. Some examples of applications include:

- Signal displays in transparent substrates with on/off functionality

- Anti-counterfeit marking for high valued commercial products (e.g. jewelry, watches)

- Product ID labeling for tracking (e.g. primary packaging in pharmaceutical industries)

The type of SwIMM produced depends on the laser processing parameters and the substrate material characteristics. The following sections describe SwIMM made in soda lime glass and in polycarbonate.

Soda lime glass is one of the most commonly used glasses in our daily life, used for windows and household glassware. Microscope slide glass (Corning 0215 type II soda lime glass) was also tested.

Polycarbonate is used in a wide range of applications where its high impact and temperature resistance, and optical properties are required. The automotive industry has started to replace glass with polycarbonate to reduce weight and improve fuel economy. Polycarbonate is also used for compact discs and DVDs. Some computer displays employ polycarbonate as well.

\subsection{Scattering SwIMM}

Since the visibility is based on scattering, the SwIMM can be illuminated from different directions. To prevent shadowing of the illumination light by laser modifications closer to the illumination source and to uniformly illuminate the entire pattern, the SwIMM lines can be written at different depths. Or the illumination light can be incident at an angle with respect to the plane of the SwIMM pattern.

However, when the transparent substrate is thinner than the divergence of the illumination light, the light will be guided by total internal reflection. In this case, the relative angle between the illumination source and the plane of the SwIMM pattern is not as critical. Thinner substrates can more tightly confine the light, making the SwIMM pattern brighter than a thicker substrate for the same luminous flux from the illumination source. Light coupling, however, can be more difficult with thinner substrates. Using a brighter illumination source increases the visibility contrast.

Two different types of scattering SwIMM are produced in glass and in polycarbonate.

\subsubsection{Scattering SwIMM in Glass}

Localized micro-cracks are formed a few tens of micrometer in size which extend radially around the larger laser-induced modification. The small size of the microcracks helps to maintain the strength of the glass under mechanical and/or thermal stress. Figure 2 shows a top view (left) of a series of SwIMM lines written in soda lime glass. The cross-section of the lines is shown in the photo on the right. The micro-cracks are very difficult to see, even in these microscope photos.

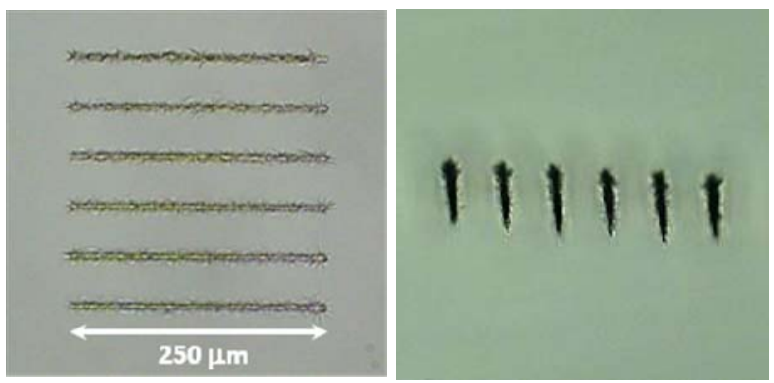

Fig. 2 Optical microscope images of the top view (left) and cross-sectional view (right) of localized micro-cracks.

In the ON state, the marks are clearly visible from a wide range of viewing angles as it is based on light scattering from micro-cracks. Figure 3 (a) shows an arrow icon in soda lime glass in its ON state (a) and OFF state (b) with the viewer at an oblique angle. 

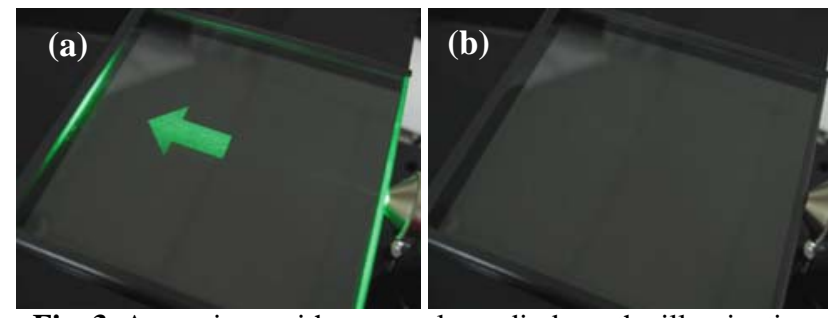

Fig. 3 Arrow icon with a green laser diode as the illumination source (a) ON, (b) OFF states. The substrate dimension is $75 \mathrm{~mm}$ x $75 \mathrm{~mm}$ [9].

When the illumination light is properly shaped and directed, multiple patterns in the same substrate can be selectively illuminated. Different signal patterns or icons can be placed in the same region.

In Figure 4, two icons (an arrow and a hazard sign) are written in the same glass substrate at different depths. In Figure 4(a), the arrow icon is illuminated from the right by a green laser diode. In Figure 4(b), the hazard icon is illuminated from the top of the image by a red laser diode. Figure 4(c) shows the OFF state without any edgeillumination.
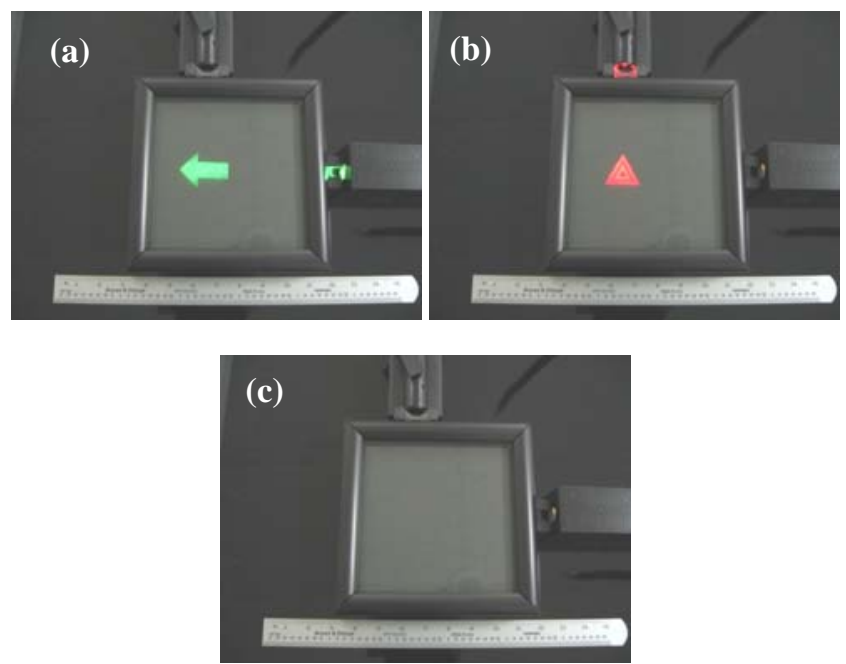

Fig. 4 (a) Green Arrow icon ON, (b) Red Hazard icon ON (c) both icons OFF. The illumination sources are green and red laser diodes. The substrate dimension is $75 \mathrm{~mm} \times 75 \mathrm{~mm}$.

\subsubsection{Scattering SwIMM in Polycarbonate}

In polycarbonate, it is not possible to create the same type of micro-crack as in glass. However, it is possible to induce a well-controlled laser modification within the polymer material by femtosecond pulses, which gives the SwIMM effect. The modified area has higher absorption of light, which suggests that the material is carbonized by rapid heating within the tightly focused laser pulses. The yield strength of polycarbonate is also quite high, which may help prevent cracking.

The dark scattering features of SwIMM marks in polycarbonate show little sign of local cracking. The size of the mark is about $5 \mu \mathrm{m}$ wide and extends up to $\sim 35 \mu \mathrm{m}$ in the laser propagation direction (Figure 5).

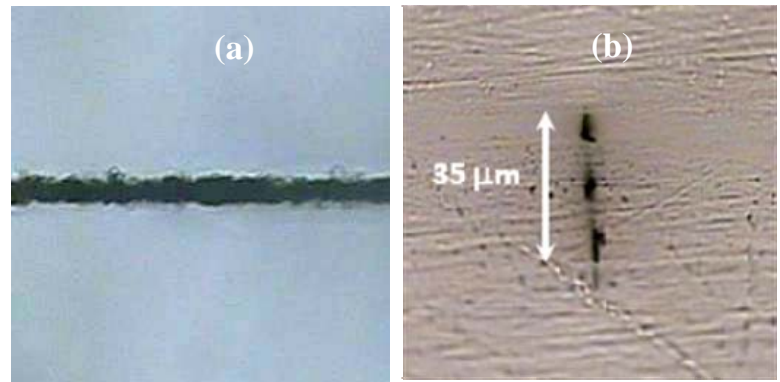

Fig. 5 Scattering SwIMM in polycarbonate, top view (a) and cross-sectional view (b) - laser incident from top in (b).

These marks have several segments along the laser propagation direction, suggesting a nonlinear re-focusing mechanism similar to filamentation $[10,11]$.

The rough laser modification is the light-scattering source. Because the modified portion of the material absorbs light more efficiently than micro-cracks, the marks appear darker in ambient lighting compared to scattering SwIMM in glass.

As was with the scattering SwIMM in glass, the SwIMM marks in polycarbonate are visible due to light scattering. So the illumination direction is not very critical. If the transparent substrate is relatively thin, the illumination can be coupled in from a side for higher ON state visibility.

Figure 6 shows an example of scattering SwIMM in polycarbonate in its ON and OFF states. The substrate is illuminated by a set of LEDs from the bottom edge of the substrate.
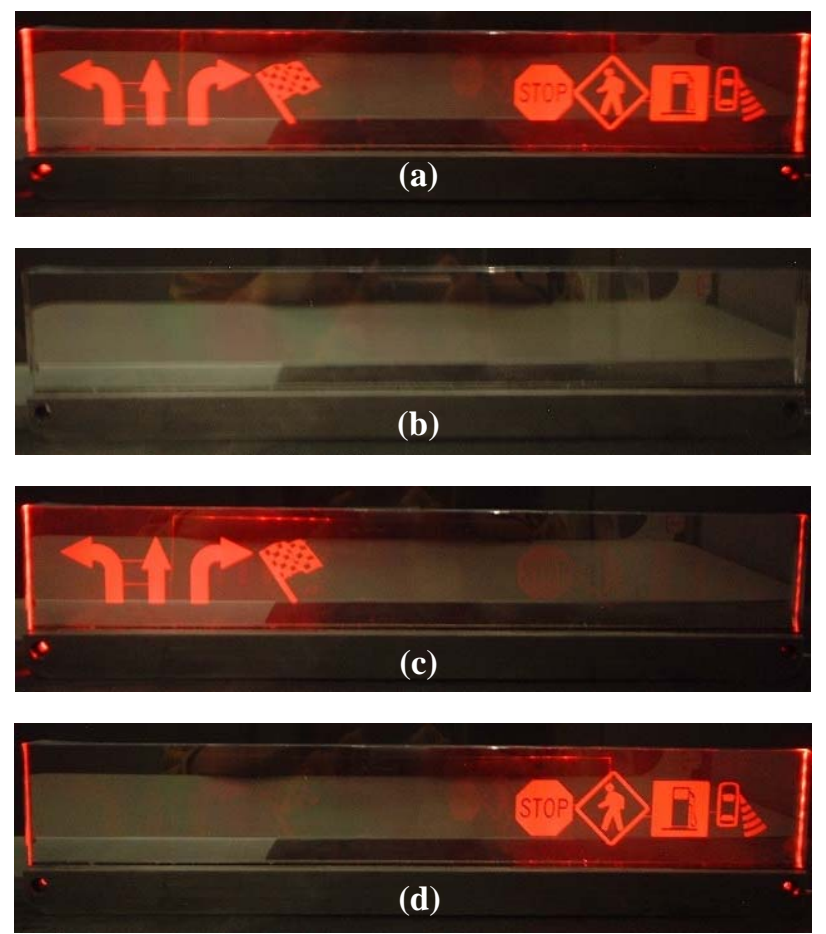

Fig. 6 Automotive icons fabricated in $3 \mathrm{~mm}$ thick polycarbonate substrate. (a) all icons ON, (b) all icons OFF, (c) left icons ON, (d) right icons ON. The width of the substrate is about $35 \mathrm{~cm}$. The illumination source is a red LED array.

By using a stack of multiple substrates, different patterns can be placed in the same field of view, with each pattern in a separate substrate with a different illumination 
source. Each pattern can be selectively illuminated since the very thin air gap between substrate layers will confine the respective illumination light to one layer by total internal reflection.

Figure 7 shows the letter " $N$ " in the top $0.75 \mathrm{~mm}$ thick polycarbonate substrate and the letter " $S$ " in the bottom substrate. In the left photo, the illumination source coupled to the top layer is turned ON, only showing the "N". In the middle photo, the illumination sources are OFF. In the right photo, the illumination source coupled to the bottom polycarbonate layer is turned ON, showing the " $\mathrm{S}$ ", but not the "N".
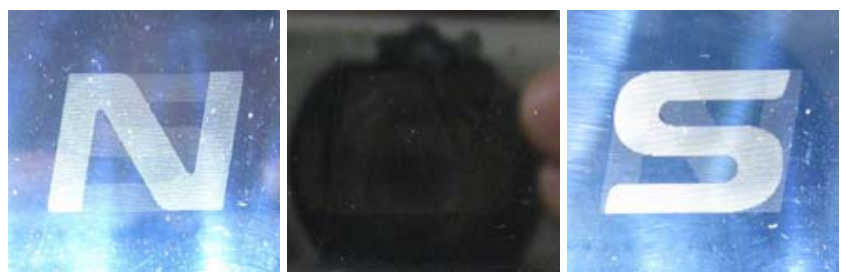

Fig. 7 Polycarbonate stack with "N" in the top layer and "S" in the bottom layer. (left: front substrate ON, center: all OFF, right: back substrate $\mathrm{ON}$ ). The width of the letters is about $2 \mathrm{~cm}$. The illumination source is a white LED array.

\subsection{Reflecting SwIMM}

Rather than localized micro-cracks, reflecting SwIMM is based on planar cracks. An SEM image of the planar crack is shown below (Figure 8).

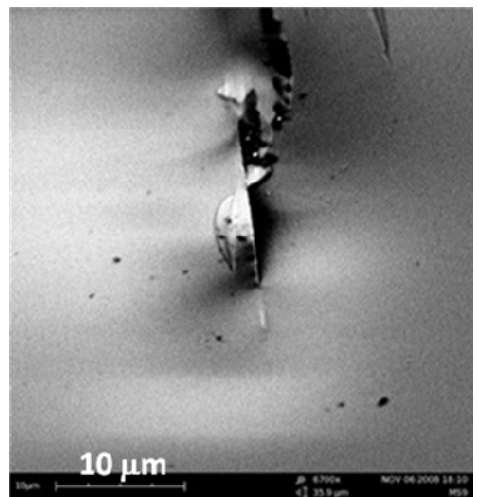

Fig. 8 SEM image of planar crack (cross-sectional view).

The planar crack is parallel to the plane defined by the laser propagation and scanning directions. It is usually accompanied by some amount of melt. The planar crack can effectively reflect the incident illumination light.

As the reflecting SwIMM visibility is based on a reflection of the illumination light by the planar crack, the viewer must be positioned correctly relative to the plane of the SwIMM mark and the illumination direction. An example of the illumination and the viewing angle combination is shown in Figure 9.

When the marks are illuminated from an edge (zero incident angle), as with scattering SwIMM, the visibility of the pattern is poor.

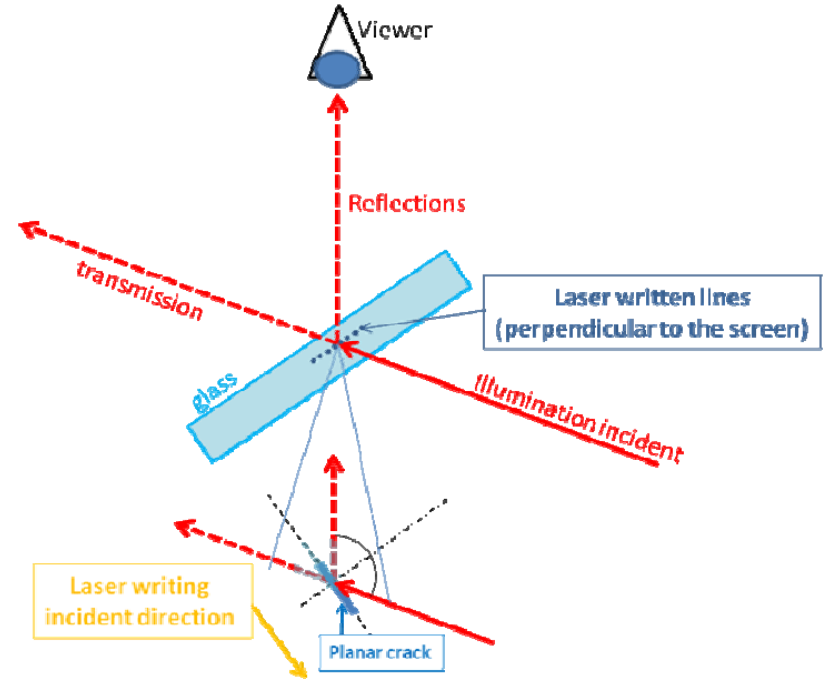

Fig. 9 Illumination and viewing angles for high visibility for reflecting SwIMM.

The visibility of the OFF state can be significantly less than scattering SwIMM in an ambient lighting condition. With the viewer in the specific angle with respect to the planar crack orientation and the illumination source, a very high visibility contrast can be achieved with the reflective marks.

An example of reflecting SwIMM mark is shown in Figure 10. In Figure 10(a), the viewer is at the optimal viewing position. In Figure 10(b), the viewer is not at the optimal viewing position. In contrast to scattering SwIMM (Figure 3(a)), appropriate illumination and viewing position are important for good visibility.
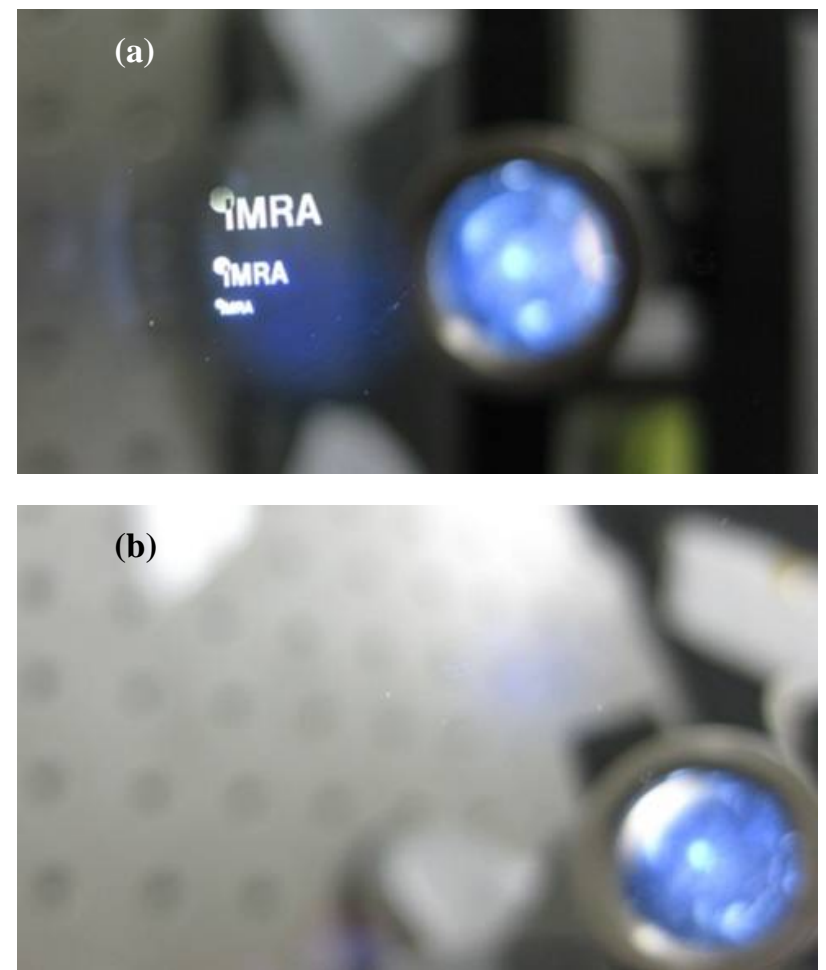

Fig. 10 Reflecting SwIMM in microscope slide glass illuminated by a flashlight (a) illumination $\mathrm{ON}$ and the viewer at the optimal visibility angle, (b) illumination $\mathrm{ON}$, but the viewer not in the optimum visibility angle. The heights of the logos are $1.5 \mathrm{~mm}$, $1.0 \mathrm{~mm}$, and $0.5 \mathrm{~mm}$. 


\section{Fabrication}

Though each material tested is optically transparent, they have different physical properties that affect the type of SwIMM mark that can be produced and the laser processing conditions required to make the mark.

\subsection{Scattering SwIMM in Glass}

To create localized micro-cracks in soda lime glass, relatively longer pulse duration (25 - 200 ps) was preferred. Scanning created ideal localized micro-cracks along the laser scanned lines. Raster-scanning was used to make the patterns.

It is possible to create multi-layer patterns since the depth of the modification is on the order of a few 10s of $\mu \mathrm{m}$. The type of material modification created was sensitive to the depth of the focus in the material.

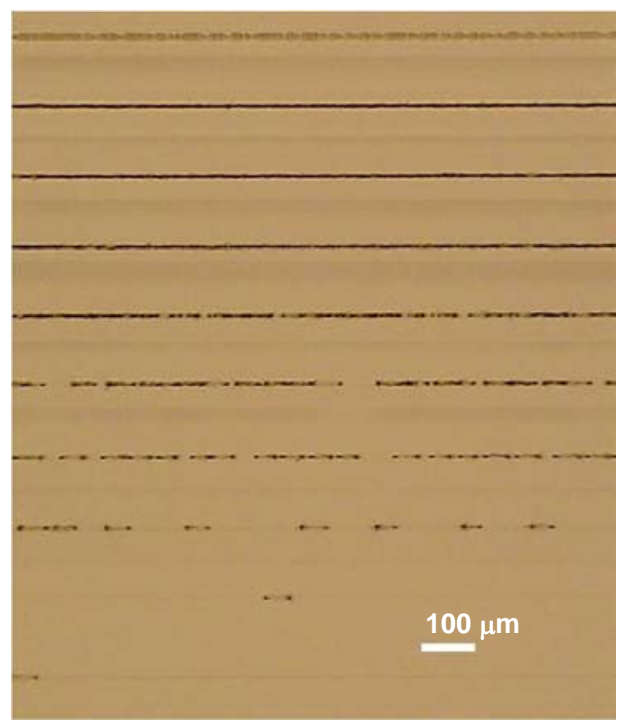

Fig. 11 Localized micro-cracks with various focus depths. (The relative depth between adjacent lines is $\sim 380 \mu \mathrm{m}$ ). The top line is at $\sim 380 \mu \mathrm{m}$ below the surface.

When the focus is shallow, the modifications appear to look more like melting, without micro-cracks. When the focus is deep, the micro-cracks became sporadic. There was a limited range of depth where the consistent localized micro-cracks were created at a particular combination of the laser process parameters.

\subsection{Reflecting SwIMM in Glass}

Reflecting SwIMM were made in a similar fashion. The process conditions were much wider than the case with micro-cracks. In microscope slide glass, 2 different pulsewidths ( $\sim 50 \mathrm{fs}, \sim 25 \mathrm{ps)}$ and 2 focusing geometries (NA0.5, NA0.55) were tested with 3 average powers and 6 scan speeds.

The strongest reflecting marks were fabricated with tightly focused ultrashort pulses. However, longer pulses ( 25 ps) and/or looser focus were also able to make reflecting marks, but with much lower reflection. The effective reflecting marks usually have a combination of melt with an extended planar crack.
When the net fluence is high (high energy, slow speed) the modification typically becomes rough in the center surrounded by a large melted region.

With looser focus (NA0.5) in the moderate net fluence range, the modification is like a waveguide with a smooth, round melt region which does not reflect or scatter the illumination light.

Scattering marks were fabricated at fast scan speeds (200 mm/s) within the lower net fluence range. With ultrashort pulses, the modification appears to be rough but fine in texture rather than micro-cracks. There was also a process window for scattering marks at low-power, lowspeed combination with longer pulse. These modifications also appear to have a fine rough texture.

\subsection{Scattering SwIMM in Polycarbonate}

To create well-controlled scattering marks in polycarbonate, ultrashort pulses were used. The average power, focusing condition and scan speed of the laser all contribute to the resultant material modifications. With a tight focus in polycarbonate, unlike the case with glass, the material modification did not depend strongly on the focus depth. However, when a looser focus was used, the depth clearly affected the density/strength of the material modification.

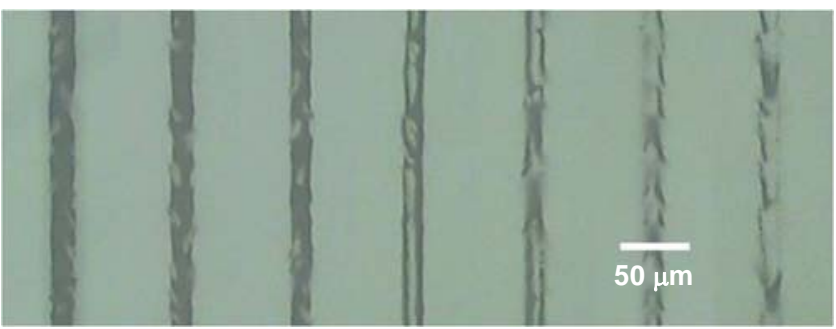

Fig. 12 Sub-surface lines of scattering material modification by looser focus at various focus depths in polycarbonate (The shallowest line (the most left) is at a depth of $\sim 75 \mu \mathrm{m}$ from the top surface. The relative depth between adjacent lines is $\sim 160 \mu \mathrm{m}$ ).

With a similar power dosage, looser focus produced a better visibility trade-off compared to tighter focus, with a much lower cumulative fluence. Figure 13 compares the SwIMM patterns with 1-W power focused by $100-\mathrm{mm}$ lens scanned at $550 \mathrm{~mm} / \mathrm{s}$ and $100-\mathrm{mW}$ power focused by 20x aspheric lens scanned at a speed of $50 \mathrm{~mm} / \mathrm{s}$. The large volume of modification produced by the looser focus probably contributes to more efficient scattering of the illumination light.

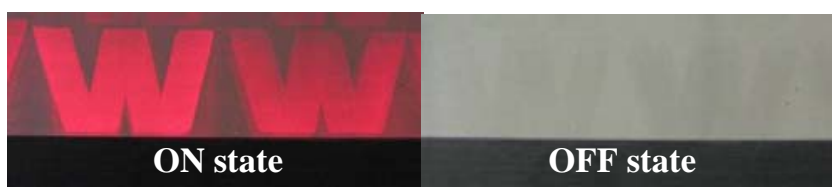

Fig. 13 Letter "W" written in 0.75 mm-thick polycarbonate. (left: looser focus, faster scan speed, right: tighter focus, slower scan speed). The width of the letters is about $2 \mathrm{~cm}$. The illumination source is a red LED array.

Higher visibility in the OFF state does not necessarily mean a higher visibility in the ON state. This is why the optimization of the visibility trade-off is very import for the marks to function well as SwIMM. 


\section{Microscopic Micro-Marking}

In addition to the SwIMM process, ultrashort pulses can be used to make sub-surface, microscopic patterns in transparent materials that require a high-magnification microscope to see. Figure 14 shows IMRA's logo written in sapphire using low energy (455 $\mathrm{nJ})$ ultrashort pulses $(<500$ fs) with tight focusing (NA 0.55). The height of each character in the logo is about $12 \mu \mathrm{m}$.

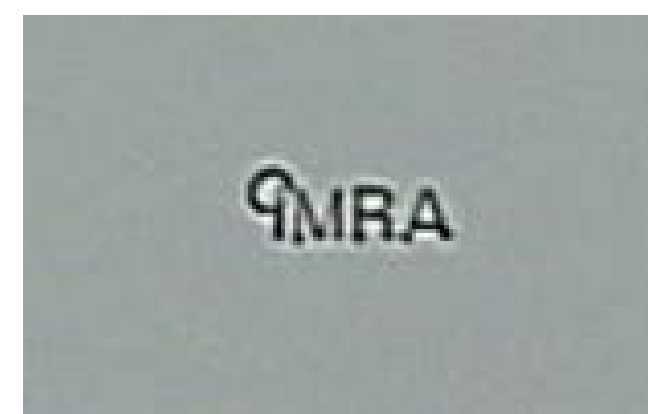

Fig. 14 IMRA logo in sapphire viewed using an optical microscope (letters are about $12 \mu \mathrm{m}$ tall).

With such a small mark, it is very difficult to find the logo and impossible to read it with the unaided eye. Even using a high power microscope, this mark is very difficult to find. High magnification is necessary to recognize the mark. But the depth of focus for a high magnification objective is very short, so it is necessary to know the depth of the mark, otherwise the mark will not be in focus. Such pattern can be used effectively for anti-counterfeit ID marking since they are difficult to find without prior knowledge of the location and also difficult to modify.

\section{Summary}

Ultrashort laser pulses enable unique material processing within transparent materials. With femtosecond pulse, high power, high repetition rate FCPA $\mu$ Jewel D-1000 fiber laser, various types of transparent materials were modified with different processes to make Switchable Inner MicroMarks (SwIMM). The high visibility contrast between ON (with external illumination) and OFF (in an ambient lighting) states enable unique controllable displays.

With the wide range of repetition rate and pulse energies of the FCPA $\mu$ Jewel, it is possible to choose the proper process condition for the necessary thermal management for each transparent material which results in the targeted material modification.
In soda lime glass, localized micro-cracks were fabricated with relatively longer pulse. In polycarbonate, a controlled material modification feature (possibly carbonization) of material was induced by the ultrashort pulse laser exposure. These 2 types of material modifications scatter illumination light efficiently in all the directions; whereas they are least visible in ambient lighting conditions. A planar crack was created in few different types of glass. A short pulse was effective to create the planar crack. As the marks become visible by reflection of the illumination light, a specific combination of illumination and viewing angles offers high visibility of the mark. Because scattering is minimum with the planar crack, a very high visibility contrast between ON and OFF states can be achieved with this feature.

Additionally, $\mu \mathrm{m}$-size marking was demonstrated in sapphire. The pattern is invisible to unaided eyes so that it can be used for anti-counterfeit applications.

\section{References}

[1] B.C. Stuart, M.D. Feit, S. Herman, A.M. Rubenchik, B.W. Shore, and M.D. Perry: Phys. Rev. B, 53, (1996) 1749.

[2] RL Sutherland: "Handbook of Nonlinear Optics, $2^{\text {nd }}$ Edition”, (CRC, New York, 2003) p.579.

[3] S. Eaton, H. Zhang, P. Herman, F. Yoshino, L. Shah, J. Bovatsek, and A. Arai: Opt. Exp., 13, (2005) 4708.

[4] T. Tamaki, W. Watanabe, and K Itoh: Jpn. J. Appl. Phys. Part 2, 44, (2005) L687-L689., T. Tamaki, W. Watanabe, and K. Itoh: Opt. Exp., 14, (2006) 10460.

[5] J. Bovatsek, A. Arai, and C.B. Schaffer: CLEO/QELS and PhAST Technical Digest, (2006) CThEE6.

[6] I. Miyamoto, A. Horn, J. Gottmann, D. Wortmann, and F. Yoshino: Journal of Laser Micro/Nanoengineering, 2, (2007) 57.

[7] J. M. Bovatsek, A. Y. Arai, F. Yoshino, and Y. Uehara: PW06 SPIE, 6102-1

[8] F. Yoshino, L. Shah, M. Fermann, A. Arai, and Y. Uehara: Journal of Laser Micro/Nanoengineering, 3, (2008) 157.

[9] James Tyrrell: Optics.org article, "Fiber laser creates hidden icons in glass" http://optics.org/cws/article/research/25176 (2006).

[10]Z. Wu, H. Jiang, L. Luo, H. Guo, H. Yang, and Q. Gong: Opt. Lett., 27, (2002) 448.

[11] J. B. Ashcom, R.R.Gattass, C. B. Schaffer, and E. Mazur: J. Opt. Soc. Am. B, 23, (2005) 2317.

(Received: July 8, 2009, Accepted: December 1, 2009) 